\title{
Parameter estimation of a hyperelastic constitutive model for the description of polyurethane foam in large deformation
}

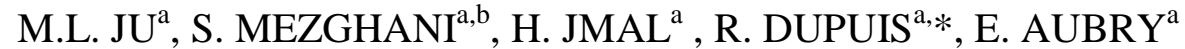 \\ ${ }^{\text {a }}$ Laboratoire MIPS, Université de Haute Alsace, 12 rue des frères Lumière, 68093 Mulhouse, FRANCE. \\ ${ }^{\mathrm{b}}$ Research Unit U2MP, National Engineering School of Sfax (ENIS), B.P 1173. 3038-Sfax- TUNISIA.
}

\begin{abstract}
Polyurethane foam is widely used in such areas as the automotive industry and sport, and in the field of packaging because of its low stiffness, high compressibility and its ability to absorb strain energy. The quasi-static behaviours of polyurethane foam are highly nonlinear and elastic. This paper demonstrates that the nonlinear elastic mechanical behaviour of compressible polyurethane foam during the loading and unloading quasi-static compression tests can be described by applying Ogden's modified model. The experimental data from a uniaxial compression of three types of polyurethane foam in three different strain rates are used for parameter identification. A nonlinear optimization method helps to ensure that the parameters are satisfied with stability conditions. Thanks to the optimized parameter results, the numerical simulations agree with the experimental data. Finally, the errors between the model results and the experimental results are analyzed and the unloading phases are discussed in detail.
\end{abstract}

Keywords: Polyurethane foam; Quasi-static behaviour; Ogden's model; Uniaxial compression; Parameter identification.

*Corresponding author. Tel.: +33 3893369 25; fax: +33 389423282

E-mail address: raphael.dupuis@uha.fr 


\section{Nomenclature}

\begin{tabular}{|c|c|c|}
\hline Symbols & Units & Definitions \\
\hline$B$ & & Left Cauchy-Green deformation tensor \\
\hline$C$ & & Right Cauchy-Green deformation tensor \\
\hline Ceil & & Round to the nearest integer \\
\hline$D$ & $\left(\sec ^{-1}\right)$ & Strain rate tensor \\
\hline$F$ & & Deformation gradient tensor \\
\hline$\left(I_{i}\right)_{i=1,2,3}$ & & $\begin{array}{l}\text { Invariants of the right Cauchy-Green deformation } \\
\text { tensor }\end{array}$ \\
\hline$J$ & & Determinant of the deformation gradient \\
\hline$k_{0}$ & (MPa) & Initial bulk modulus \\
\hline$L \times w \times h$ & $(\mathrm{~mm} \times \mathrm{mm} \times \mathrm{mm})$ & Initial dimensions of polyurethane foam samples \\
\hline$N$ & & Minimum number of test samples \\
\hline$N_{c y c}$ & & Number of cycles in quasi-static tests \\
\hline$R$ & & A proper orthogonal tensor \\
\hline$S$ & $(\mathrm{MPa})$ & Cauchy stress tensor \\
\hline$T$ & $(\mathrm{sec})$ & Test period \\
\hline$T_{\text {ech }}$ & $(\mathrm{sec})$ & Sampling period \\
\hline$U$ & & Right stretch tensor \\
\hline$V$ & & Left stretch tensor \\
\hline$W$ & & Strain energy function \\
\hline$\left(\alpha_{i}\right)_{i=1,2,3}$ & & Parameters of Ogden's model \\
\hline$\left(\beta_{i}\right)_{i=1,2,3}$ & & Parameters of Ogden's model \\
\hline$\varepsilon$ & $\left(\mathrm{mm} \mathrm{mm}^{-1}\right)$ & Strain \\
\hline$\varepsilon_{0}$ & $\left(\mathrm{~mm} \mathrm{~mm}^{-1}\right)$ & Initial strain \\
\hline$\varepsilon_{\max }$ & $\left(\mathrm{mm} \mathrm{mm}^{-1}\right)$ & Maximum strain \\
\hline$\dot{\varepsilon}$ & $\left(\sec ^{-1}\right)$ & Strain rate \\
\hline$\left(\lambda_{i}\right)_{i=1,2,3}$ & & Principal stretches \\
\hline$\left(\mu_{i}\right)_{i=1,2,3}$ & $(\mathrm{MPa})$ & Parameters of Ogden's model \\
\hline$\mu_{0}$ & $(\mathrm{MPa})$ & Initial shear modulus \\
\hline$\rho_{0}$ & $\left(\mathrm{~kg} / \mathrm{m}^{3}\right)$ & Density of material in reference configuration \\
\hline$\rho$ & $\left(\mathrm{kg} / \mathrm{m}^{3}\right)$ & Density of material in deformed configuration \\
\hline$\sigma$ & $(\mathrm{MPa})$ & Stress \\
\hline
\end{tabular}




\section{Introduction}

Today, polymeric foam materials, such as polystyrene (PS), expanded polypropylene (EPP) and polyurethane (PU), are widely used in numerous industrial applications in engineering, sport, medical care. Polyurethane foams are cellular materials characterized by the spectrum of mechanical properties ${ }^{[1]}$ such as: low stiffness, low Poisson rate, low density (less than $80 \mathrm{~kg}^{-3} \mathrm{~m}^{-3}$ for flexible foam), the ability to absorb the strain energy, high compressibility and slow recovery rate. These properties help to improve the comfort of car seats ${ }^{[2]}$.

Polyurethane foams can be categorized as open or closed cell materials depending on the shape and connectivity of the cells. If foams allow fluids to flow through the cellular structure, these foams are called open-cell foams ${ }^{[3]}$. There is a great variety of open-cell foams; however, their mechanical behavior is not fully understood. Therefore, the present study aims to investigate open-cell foams thoroughly.

There are several standards for foam characterization, such as the American standard D3574-95 of the American Society for Testing Material ${ }^{[1]}$, and for methods and tests for the assessment of foam properties. The tests include those of the indentation force deflection, the ball rebound, the compression force deflection and the dynamic such as transmissivity and impact. There are also four types of foam studies ${ }^{[1]}$ : static behaviour, quasi-static behaviour, dynamic behaviour and fatigue behaviour. A more detailed description of foam behaviour can be found in ${ }^{[4,5]}$.

The polyurethane foam stress-strain response shows very large strains with a strongly nonlinear behaviour which can be described by a number of hyperelastic models based on the definition of different strain energy functions. In the literature, there are numerous models designed to fit experimental results for hyperelastic materials. Mooney proposed a model with two parameters ${ }^{[6]}$. The Neo-Hookean model described by Trelor has only one material parameter ${ }^{\text {[7] }}$, but this model was proved to be a special case of the Mooney model. In 1950, Rivlin modified the Mooney model and obtained a general expression so-called Mooney-Rivlin model ${ }^{[8,9]}$. Yang and Shim ${ }^{[10]}$ proposed a visco-hyperelastic model for foams under strain rates to capture the three-dimensional large compression behaviour. The Blatz-Ko model ${ }^{[11]}$ is also used to describe the properties of hyperelastic materials. In 1972, Ogden ${ }^{[12,13]}$ proposed a strain energy function expressed in terms of principal stretches, which is a very general expression for describing hyperelastic materials. There is an excellent correspondence between the Ogden model data and Treloar's experimental data. Other models include those of Yeoh ${ }^{[14]}$, Beatty ${ }^{[15]}$, Arruda-Boyce ${ }^{[16]}$, Bischoff et al. ${ }^{[17]}$, and Attard ${ }^{[18]}$. Numerous studies have been carried out to solve non-linear problems with the finite element method. A very detailed review on finite element formulation for non-linear analysis has been provided by Sussman and Bathe ${ }^{[19]}$. Many models are now available in commercial FEM software, such as ANSYS and ABAQUS.

Conventionally, the determination of material parameters is based on the use of test samples with a standardized geometry under a simplified strain state. Then the unknown model parameters are obtained using curve fittings from experimental data. For polyurethane foams, a wide range of tests have been used (e.g. compression, shear, and volumetric tests) in the literature to predict these parameters. These methods normally require large numbers of tests and samples with well-defined geometries. Seat polyurethane foams are mainly loaded through a compressive force. For these two reasons, numerous compression tests were performed to obtain a sufficient number of experimental results for the parameter identification of the polyurethane foams presented here. There are few articles on the mechanical behaviour of polyurethane foam, especially on the analysis of the 
unloading phase. Smardzewski et al. ${ }^{[20]}$ used a hyperelastic model to determine the elastic properties of polyurethane foams in compression tests with a loading phase. Zhang et al. ${ }^{[21]}$ used a pseudo elastic model to model the polymeric foam mechanical properties in the loading and unloading phases.

This paper describes both the loading and unloading phases and explains in detail the unloading phase affected by residual stress. The purpose of this paper is to only model the elastic behaviour of polyurethane foam with high compressive deformation and to estimate the model parameters to allow a good correlation between the model and the experimental results. The corresponding identification errors are also taken into account to analyze the results. This paper is organized as follows: the experimental conditions are presented in Section 2 which is followed by a description of the experimental details. The Ogden model is described in Section 3; this model is then used to estimate the mechanical behaviour of polyurethane foam with the experimental data. Finally, the experimental data and the model results are discussed and compared in Section 4 and conclusions are summarized in Section 5.

\section{Experimental}

In order to investigate the stress-strain relation, a series of loading-unloading uniaxial compression experiments were carried out at a constant temperature of $25^{\circ} \mathrm{C}$. The three types of polyurethane foams, designated by foam Type A, Type B and Type C, have characteristics similar to those of automotive seat foam. The properties of the three type foams are summarized in Table 1. Test specimens of polyurethane foam were cut from a block of foam $(2000 \mathrm{~mm} \times 1200 \mathrm{~mm} \times 75 \mathrm{~mm})$ obtained through expansion in a free open mould. All specimens had the same mechanical and environmental histories. They are original specimens and each specimen was compressed only one time. Contrary to Belouettar et al. ${ }^{[22]}$ and White et al. ${ }^{[23]}$ who used foam cubes cut from bolsters of car seat cushions, the type of foam chosen here helps to provide a substantially homogeneous material and isotropic, repeatable specimens ${ }^{[24]}$.

Table 1. Chemical and morphological foam characteristics

\begin{tabular}{|l|l|l|l|}
\hline & Type A & Type B & Type C \\
\hline Foam type & $\begin{array}{l}\text { Flexible polyurethane } \\
\text { foam }\end{array}$ & $\begin{array}{l}\text { Flexible polyurethane } \\
\text { foam }\end{array}$ & $\begin{array}{l}\text { Flexible polyurethane } \\
\text { foam }\end{array}$ \\
\hline Isocynate & Toluene diisocynate TDI & Toluene diisocynate TDI & Toluene diisocynate TDI \\
\hline Polyol & Polyether & Polyether & Polyether \\
\hline Expansion gas & $C O_{2}$ & $C O_{2}$ & $C O_{2}$ \\
\hline Fabrication process & Free rise & Free rise & Free rise \\
\hline Density & $28 \mathrm{~kg} / \mathrm{m}^{3}$ & $40 \mathrm{~kg} / \mathrm{m}^{3}$ & $50 \mathrm{~kg} / \mathrm{m}^{3}$ \\
\hline Average cell size & $828 \mu \mathrm{m}$ & $941 \mu \mathrm{m}$ & $633 \mu \mathrm{m}$ \\
\hline Samples shape & cubic & cubic & cubic \\
\hline $\begin{array}{l}\text { Dimensions } \\
(\text { L } \times w \times h)\end{array}$ & $75 \mathrm{~mm} \times 75 \mathrm{~mm} \times 75 \mathrm{~mm}$ & $75 \mathrm{~mm} \times 75 \mathrm{~mm} \times 75 \mathrm{~mm}$ & $75 \mathrm{~mm} \times 75 \mathrm{~mm} \times 75 \mathrm{~mm}$ \\
\hline Cell type & open & open & open \\
\hline
\end{tabular}

All the tests were performed on a usual compression-tension testing device INSTRON 33R4204 driven with BLUEHILL software (Figure 1). This device includes a basis frame and an upper block 
which moves vertically. Two $150 \mathrm{~mm}$ diameter compression plates were installed: one on the base of the machine and the other on the force sensor of the crosshead. The two plates were checked to be strictly parallel. Before starting the tests, the top plate had to move down slightly for full contact with the material because the top and bottom of each foam samples were not exactly parallel. In order to establish a homogeneous deformation field, the shear stresses between the two plates on the top and bottom sides and the test specimen at the uniaxial compression test had to be eliminated ${ }^{[25]}$. To minimize the noise contribution, the maximum experimental response force of foam had to be slightly less than the load cell maximum capacity. All the test conditions including the strain rate, the maximum compression level, the number of cycles, the sampling period, and the test mechanical parameters exported were conducted using the BLUEHILL software configuration window.

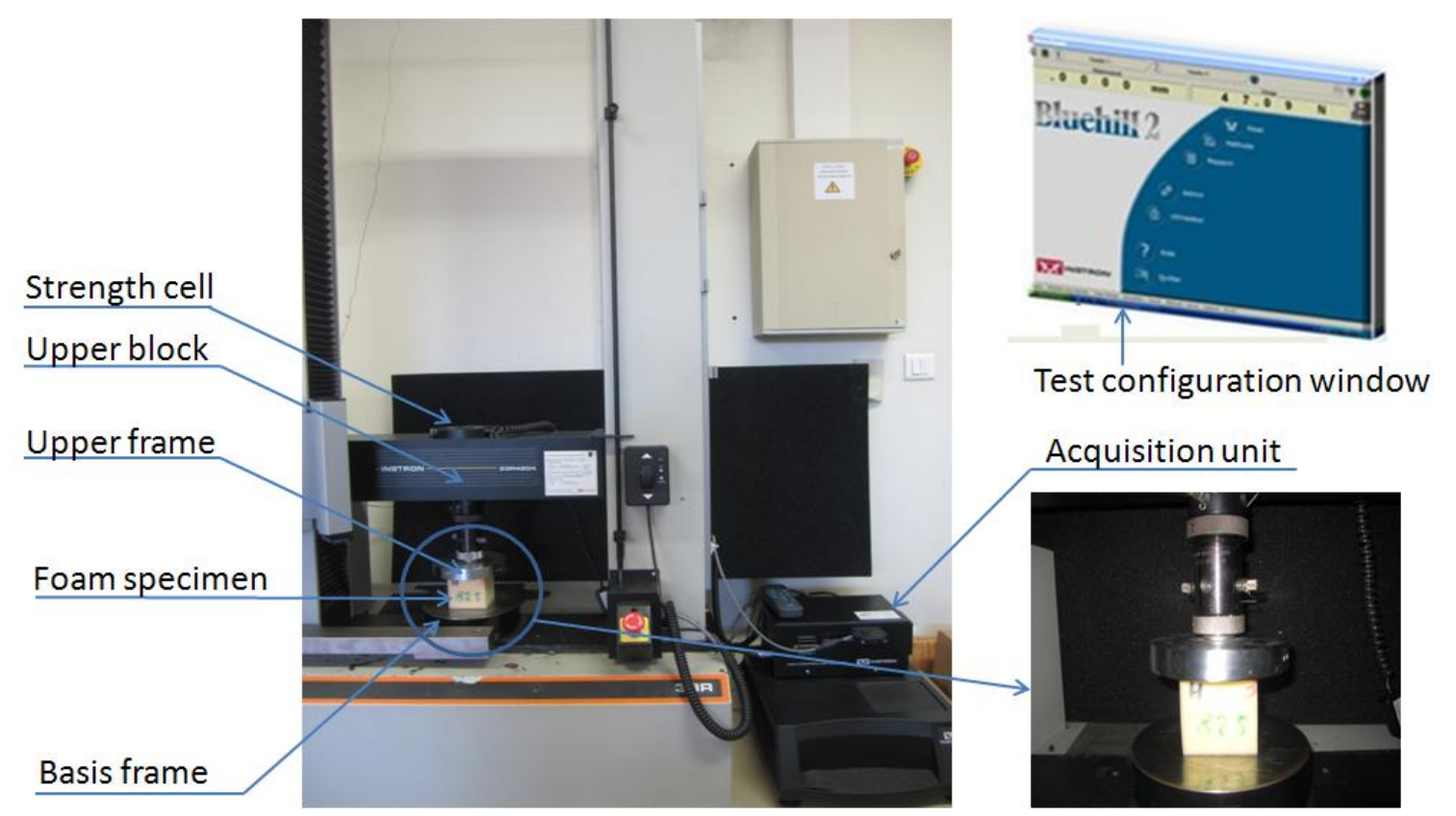

Fig. 1. Compression test device

The three types of polyurethane foam were initially put between the upper frame and the basis frame of the machine. The test started when the upper frame affected the foams and then the upper block moved down to compress the samples to the final position. The final compression ratio was $80 \%$ of the original thickness. At the end of the loading phase, the upper block changed direction and returned to the initial level. Each specimen had been quasi-statically loaded and then unloaded with a constant speed during the test process. The conditions of all tests are given in Table 2 .

Table 2. Quasi-static compression test conditions

\begin{tabular}{|c|c|c|c|c|c|c|c|}
\hline & & $\mathrm{N}_{\text {cyc }}$ & $\dot{\varepsilon}\left(\sec ^{-1}\right)$ & $\varepsilon_{0}(\%)$ & $\varepsilon_{\max }(\%)$ & $T(\mathrm{sec})$ & $T_{e c h}(\mathrm{sec})$ \\
\hline \multirow{3}{*}{$\begin{array}{l}\text { Foam } \\
\text { A B C }\end{array}$} & Test 1 & 1 & $1.0610^{-2}$ & 0 & 80 & 150 & 0.0625 \\
\hline & Test 2 & 1 & $5.3310^{-3}$ & 0 & 80 & 300 & 0.125 \\
\hline & Test 3 & 1 & $6.6610^{-4}$ & 0 & 80 & 2400 & 2 \\
\hline
\end{tabular}

At the start, 15 test samples of each strain rate for each foam were taken for the preliminary test. Then, the minimum numbers of test samples which were determined to ensure the statistical quality of the parameters were calculated using equation (20) and they are summarized in Table 3. More details are given in the discussion section. 
Table 3. Minimum of $\mathrm{N}$ test samples

\begin{tabular}{|l|l|l|l|}
\hline $\mathrm{N}$ & Test 1 & Test 2 & Test 3 \\
\hline Foam A & 17 & 37 & 10 \\
\hline Foam B & 17 & 37 & 17 \\
\hline Foam C & 15 & 26 & 17 \\
\hline
\end{tabular}

\section{Modelling}

\subsection{Constitutive Theory}

According to the empirical results, polyurethane foams show large strains, highly non-linear elastic and some inelastic properties. This study is restricted to the elastic properties of polyurethane foam and their stress-strain relationship can be characterized by a strain energy function which is related to the principal stretches. Hyperelastic constitutive models are adapted for this description ${ }^{[25]}$. There are some basic equations for hyper elasticity theories. Polymeric foam is mainly regarded as a continuous body. Consider a particle or a material point with a position vector $\mathrm{X}$ in the undeformed configuration relative to an arbitrarily chosen origin. After a displacement of the body, the new position of the particle is given by the vector position x. For simplicity's sake, consider Cartesian coordinate systems and let $\mathrm{X}$ and $\mathrm{x}$ have coordinates $X_{\alpha}$ and $x_{i}$, where $\alpha, i \in\{1,2,3\}$, so, the deformation gradient tensor $\mathrm{F}$ is defined by ${ }^{[26]}$ :

$$
F=\operatorname{Grad}(x)=\frac{\partial x}{\partial X}
$$

According to the polar decompositions, the deformation gradient can be decomposed into a product of two second-order tensors: an orthogonal tensor and a positive definite symmetric tensor, i.e.

$$
F=R U=V R
$$

where the tensor $\mathrm{R}$ is a proper orthogonal tensor, representing a rotation; the tensor $\mathrm{U}$ and $\mathrm{V}$ are positive definite and symmetric, respectively the right and left stretch tensors. The deformation can be described as the left Cauchy-Green deformation tensor:

$$
B=V^{2}=F \cdot F^{T}
$$

Or the right Cauchy-Green deformation tensor:

$$
C=U^{2}=F^{T} \cdot F
$$

The invariants of $\mathrm{C}$ and $\mathrm{B}$ are often used in the expressions for strain energy density functions. If the principal stretches are denoted by $\lambda_{i}$, then the most common quoted triad of invariants are given by:

$$
\begin{aligned}
& I_{1}=\operatorname{tr}(C)=\lambda_{1}{ }^{2}+\lambda_{2}{ }^{2}+\lambda_{3}{ }^{2} \\
& I_{2}=\frac{1}{2}\left((\operatorname{tr} C)^{2}-\operatorname{tr}\left(C^{2}\right)\right)=\lambda_{1}{ }^{2} \lambda_{2}{ }^{2}+\lambda_{1}{ }^{2} \lambda_{3}{ }^{2}+\lambda_{2}{ }^{2} \lambda_{3}{ }^{2} \\
& I_{3}=\operatorname{det}(C)=\lambda_{1}{ }^{2} \lambda_{2}{ }^{2} \lambda_{3}{ }^{2}
\end{aligned}
$$

A strain energy function can represent the stress-strain behaviour of hyperelastic materials and the stress tensor can be generated by the derivation of the strain energy function with regard to the strain tensor. Therefore, the basis equation of mechanical energy is expressed as:

$$
\dot{W}=J S \cdot D
$$

where $\mathrm{W}$ is the strain energy function, $J=\operatorname{det} F$ is the determinant of the deformation gradient and $\mathrm{S}$ is the Cauchy stress tensor. $\mathrm{D}$ is the strain rate tensor with the right Cauchy-Green tensor $\mathrm{C}$ 
and given by the following equation:

$$
D=\frac{1}{2} F^{-T} \cdot \dot{C} \cdot F^{-1}
$$

From the principle of objectivity, W must be a non-negative tensor function of the right CauchyGreen tensor C.

$$
W=W(C)= \begin{cases}>0 & \text { for } \mathrm{C} \neq \mathrm{I} \\ =0 & \text { for } \mathrm{C}=\mathrm{I}\end{cases}
$$

The strain energy W is always zero in the reference configuration $(C=I)$ and for the current configuration $(C \neq I)$ the strain energy always has to be non-negative.

Considering the results of Green and Adkins ${ }^{[27]}$, Equation (8) is inserted into (6) by considering (7) results in the most general structure of the constitutive equation for non-linear, hyperelastic, isotropic material behaviour:

\subsection{Ogden's model}

$$
S=\frac{1}{2} J^{-1} F \cdot \frac{\partial W(C)}{\partial C} \cdot F^{T}
$$

From a phenomenological standpoint several attempts have been made to obtain a realistic mathematical explanation of the mechanical behaviour of highly elastic materials. The model developed by Ogden ${ }^{[12]}$ is widely used for incompressible materials and the stored energy, W, is expressed in the form of a series:

$$
W=\sum_{n} \frac{\tilde{\mu}_{n}}{\alpha_{n}}\left(\lambda_{1}^{\alpha_{n}}+\lambda_{2}^{\alpha_{n}}+\lambda_{3}^{\alpha_{n}}-3\right)
$$

where $\tilde{\mu}_{n}, \alpha_{n}$ are parameters, $\mathrm{n}$ is the number of terms in the series. To represent all three types of strain, a three-term expression is required ${ }^{[28]}$. According to Equation (10), the principal Cauchy stresses are simply

$$
\sigma_{i}=\sum_{n} \tilde{\mu}_{n} \lambda_{i}^{\alpha_{n}}-P
$$

where $\lambda_{i}$ is the principal stretches and given by $\lambda_{i}=1+\varepsilon_{i}$, where $\varepsilon_{i}$ are strains (for compression $\left.\varepsilon_{i}<0\right)$ and $\mathrm{P}$ is an arbitrary hydrostatic pressure introduced because of the incompressibility constraint.

For the sake of describing the mechanical behaviour of highly compressible polyurethane foams, the modified Ogden model will be used and the strain energy potential is represented as ${ }^{[13,29,30]}$

$$
W=\sum_{i=1}^{N} 2 \frac{\mu_{i}}{\alpha_{i}^{2}}\left(\left(\lambda_{1}^{\alpha_{i}}+\lambda_{2}^{\alpha_{i}}+\lambda_{3}^{\alpha_{i}}-3\right)+f(J)\right)
$$

where $\mu_{i}, \alpha_{i}$ are material parameters, $f(J)$ is the volumetric function and $\mathrm{J}$ is the determinant of the deformation gradient. It can be denoted as:

$$
J=\frac{\rho_{0}}{\rho}=\lambda_{1} \lambda_{2} \lambda_{3}
$$

where $\rho_{0}, \rho$ are the density in reference and deformed results respectively.

In the present paper, this model is used to determine the mechanical behaviour of polyurethane foams. A possible form of the volumetric function $f(J)$ is given by Storakers ${ }^{[30]}$ 


$$
f(J)=\frac{1}{\beta_{K}}\left(J^{-\alpha_{K} \beta_{K}}-1\right)
$$

where $\beta_{k}$ are additional material parameters. For incompressible materials, $J=1$. So, $f(J)=0$ and considering $\mu_{i}=\frac{\tilde{\mu}_{i} \alpha_{i}}{2}$, (12) will be reduced to (10).

The initial shear modulus $\mu_{0}$ and bulk modulus $k_{0}$ are given by:

$$
\mu_{0}=\sum_{i=1}^{N} \mu_{i} \text { and } \mathrm{k}_{0}=\sum_{i=1}^{N} 2\left(\frac{1}{3}+\beta_{i}\right) \mu_{i}
$$

Equation (12) helps to obtain the spectral representation of (9) with the eigenvalues $\lambda_{i}$ of the right stretch tensor $\mathrm{U}$ and the eigenvectors $n_{i}$ of the left stretch tensor $\mathrm{V}$.

$$
S=\frac{1}{2} J^{-1} \sum_{i=1}^{3} \sum_{k=1}^{N}\left\{\frac{\mu_{k}}{\alpha_{k}}\left[\lambda_{i}^{\alpha_{k}}+\frac{1}{\alpha_{k}} J \frac{\partial f(J)}{\partial J}\right] n_{i} n_{i}\right\}
$$

According to Ogden ${ }^{[12,13]}$, Hill and Chia-Shun ${ }^{[29]}$ and Storakers ${ }^{[30]}$, sufficient stability conditions of the model are determined by parameters $\mu_{i}, \alpha_{i}$ and $\beta_{i}$ :

$$
\mu_{i} \alpha_{i}>0 \text { and } \beta_{i}>-\frac{1}{3}
$$

If the specimen is loaded only in a uniaxial compression, then according to (13), (14) and (16), the stress state can be deduced:

$$
\sigma_{i i}=2\left(\lambda_{i}\right)^{-1} \sum_{k=1}^{N} \frac{\mu_{k}}{\alpha_{k}}\left(\lambda_{i}^{\alpha_{k}}-J^{-\alpha_{k} \beta_{k}}\right)
$$

\subsection{Parameter Optimization}

To identify the parameters, the optimization methods are used as basic tools. The value of stressstrain experimental data can be obtained from the uniaxial compression test described in Section 2. Then the Ogden model (12) helps to calculate the model results which are compared with the experimental data. The mean square error between the experimental results and model results can be interpreted using a function $f$ of the following form:

$$
f=\frac{1}{n} \sqrt{\sum_{i=1}^{n}\left[\sigma_{m}\left(\mu_{i}, \alpha_{i}, \beta_{i}\right)-\sigma_{e}\left(\varepsilon_{e}\right)\right]^{2}}
$$

where $\sigma_{m}\left(\mu_{i}, \alpha_{i}, \beta_{i}\right)$ are the model stress results with the parameters $\mu_{i}, \alpha_{i}$ and $\beta_{i}$ and $\sigma_{e}\left(\varepsilon_{e}\right)$ are the experimental results. The objective of the optimization is to find the best combination of model parameters which minimize the function $f$. In this study, function $f$ is nonlinear, therefore a nonlinear optimization method has been used for minimization.

There are a deterministic and a random method for optimization ${ }^{[1]}$. The trust region reflective, Levenberg-Marquardt and Gradient methods are three examples of the deterministic methods which are effective when the objective function (function to optimize) changes rapidly. For the nondifferentiable and fractal and noisy functions, the random methods are a good choice and these methods include the Carlo, Nelder-Mead algorithm and the genetic algorithm, etc. In order to find the minimum results, the authors used the optimization tool in MATLAB with the solver FMINCON (Constrained nonlinear minimization). The best parameters must be chosen to minimize the least mean square error between experimental and analytical data and satisfy the boundary conditions using this solver with tight stopping criteria. 


\section{Results and Discussion}

\subsection{Experimental results}

Figure 2 shows the experimental stress-strain results for three types of foam (foam A, foam B and foam $\mathrm{C}$ ) in test 1 .

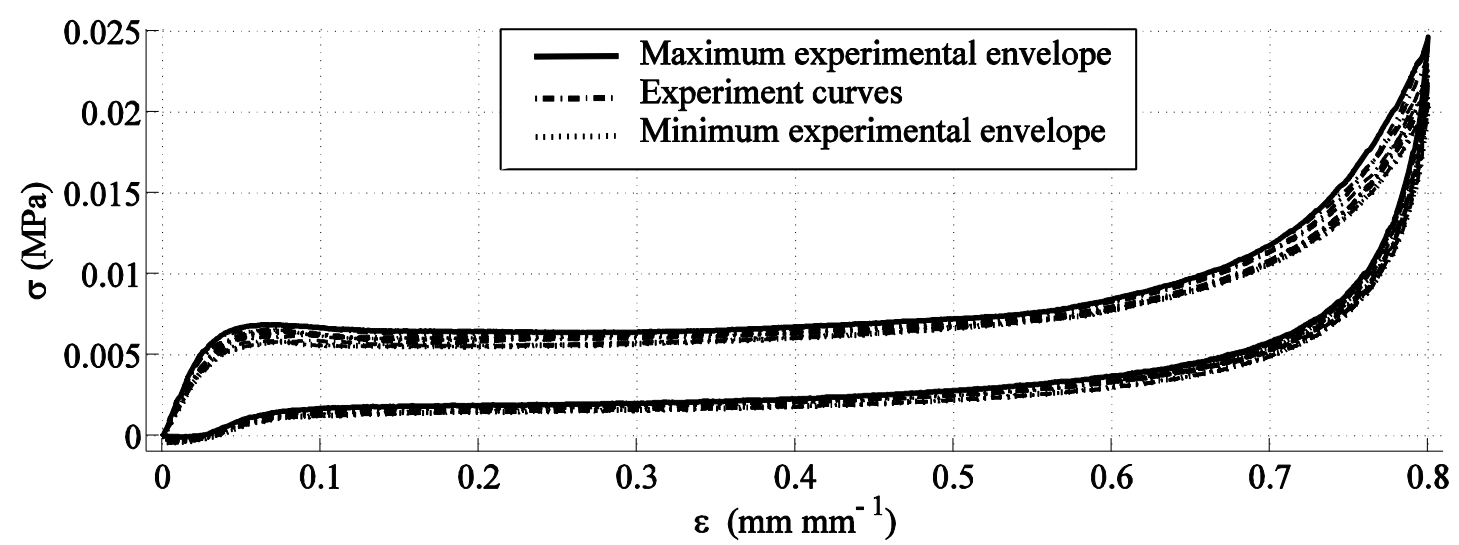

a.

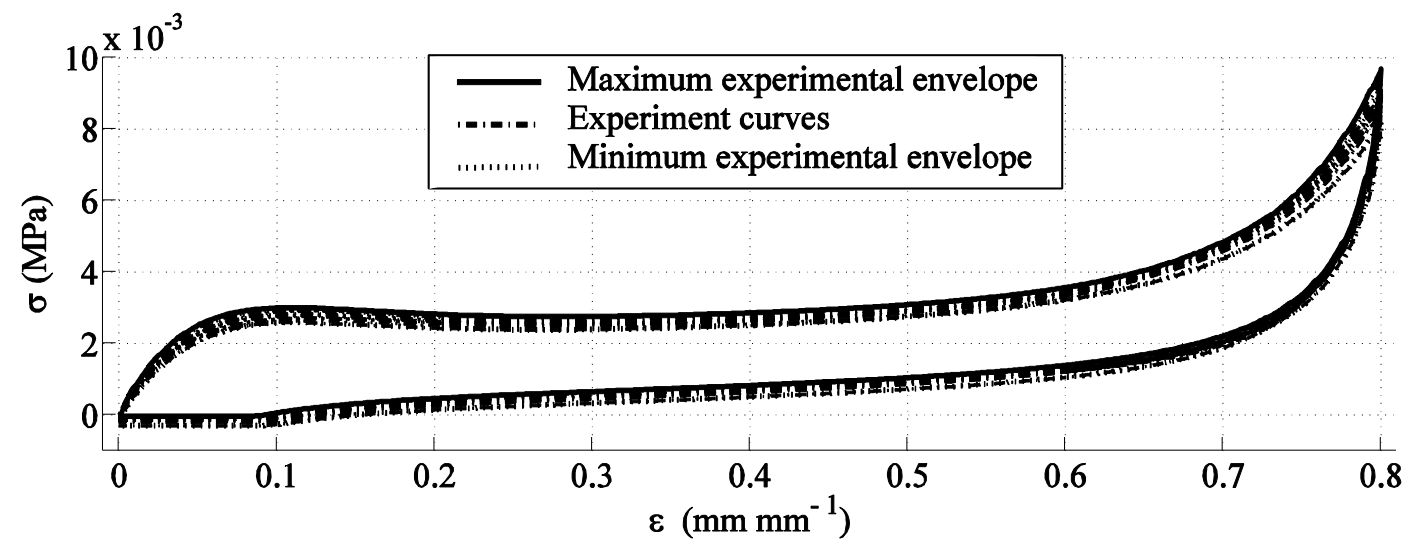

b.

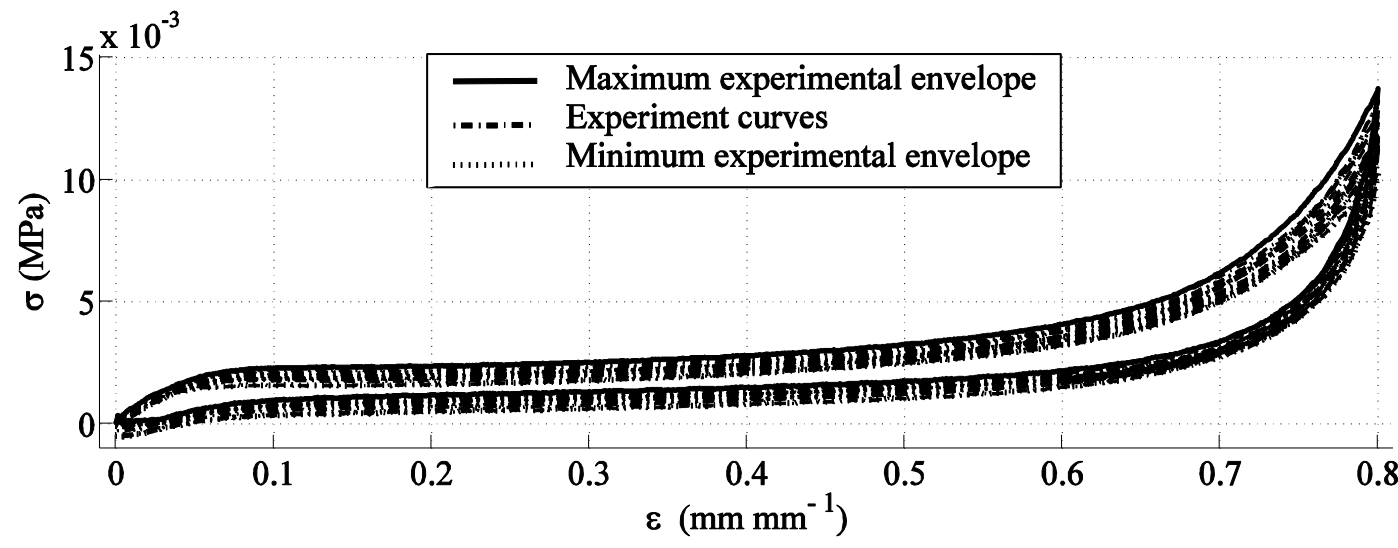

c.

Fig.2. Experimental results with strain rate $\dot{\varepsilon}=1.0610^{-2} \mathrm{sec}^{-1}$ (test1). a: foam A; b: foam B; c: foam C

As can be seen in figure 2, polyurethane foam deformation in uniaxial compression presents three stages: initial elastic deformation, collapse deformation and compaction deformation. In the first stage, the polyurethane foam deforms in a linear elastic manner due to cell wall bending, which accounts for $1 \%$ of the entire deformation. In the second stage, there is a plateau of deformation at almost constant stress. Cell walls, like thin tubes or plates, lose their stability and cause large deformations. In this stress plateau phase, the polyurethane foam undergoes large compressive 
strains and absorbs a considerable amount of specific energy. In the third stage, a region of densification occurs, where the cell walls crush together, resulting in a rapid increase of compressive stress. It can also be seen from the figure that there are large differences in the stress corresponding to the same strain level under the loading and the unloading processes. Figure 3 indicates the stress-strain curves of Foam A in three different strain rates (test 1, test 2 and test 3 ). The experimental curves between the loading phase and unloading phase are different. This means that the model parameters for loading and unloading phases should be calculated separately.

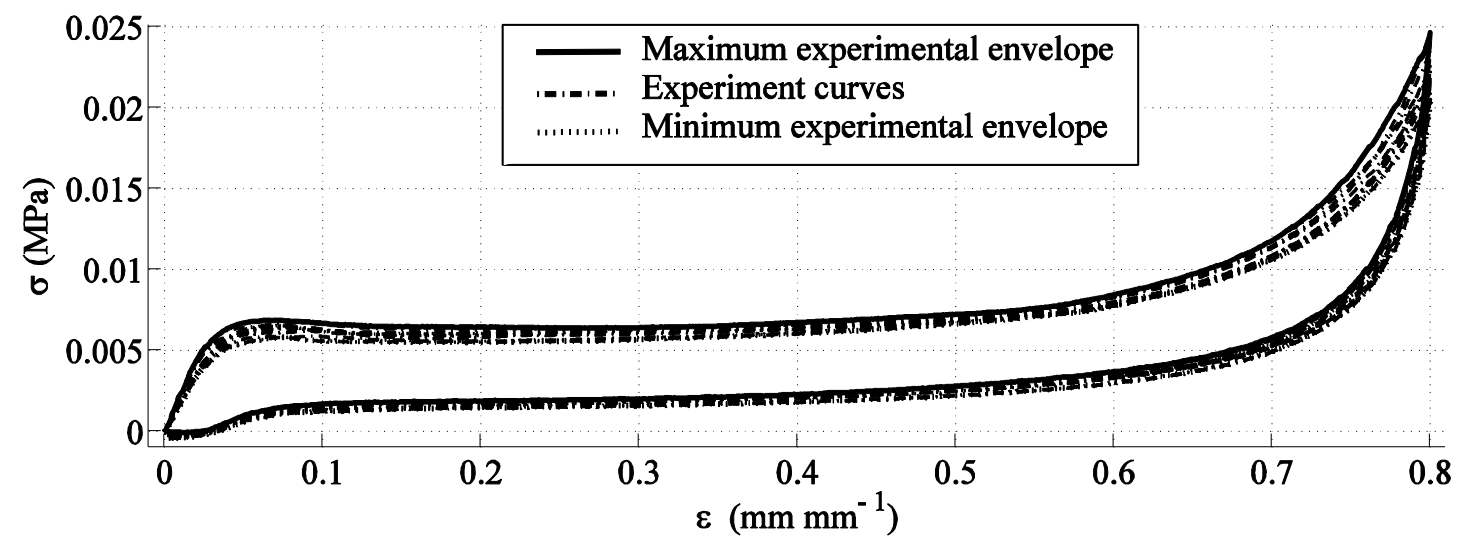

a.

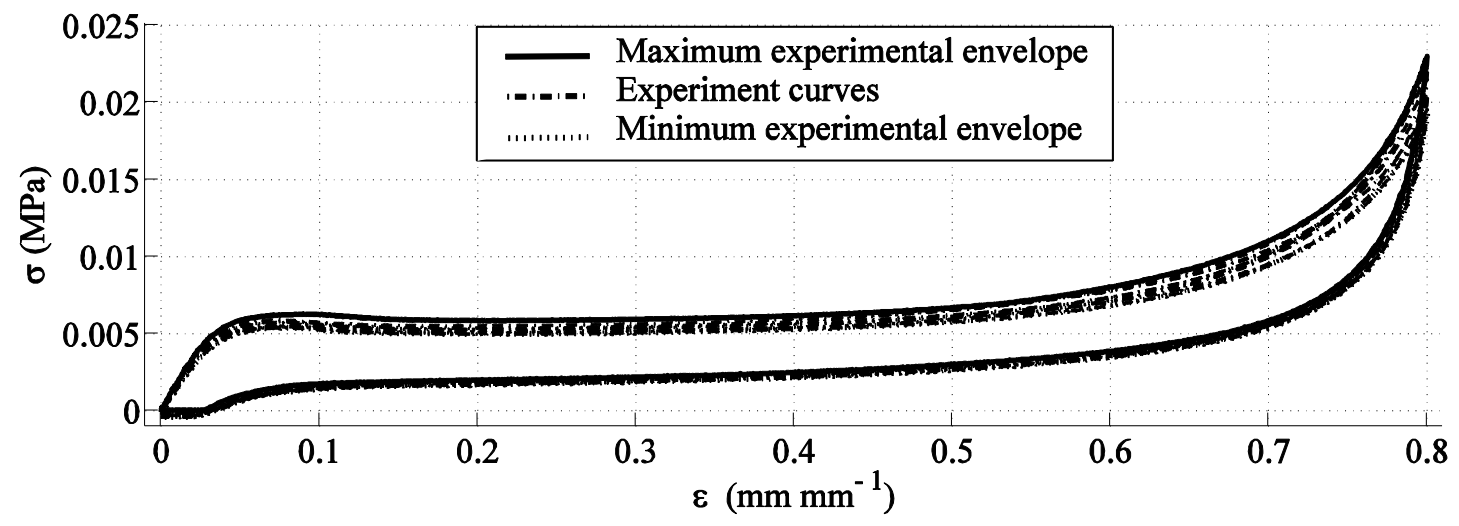

b.

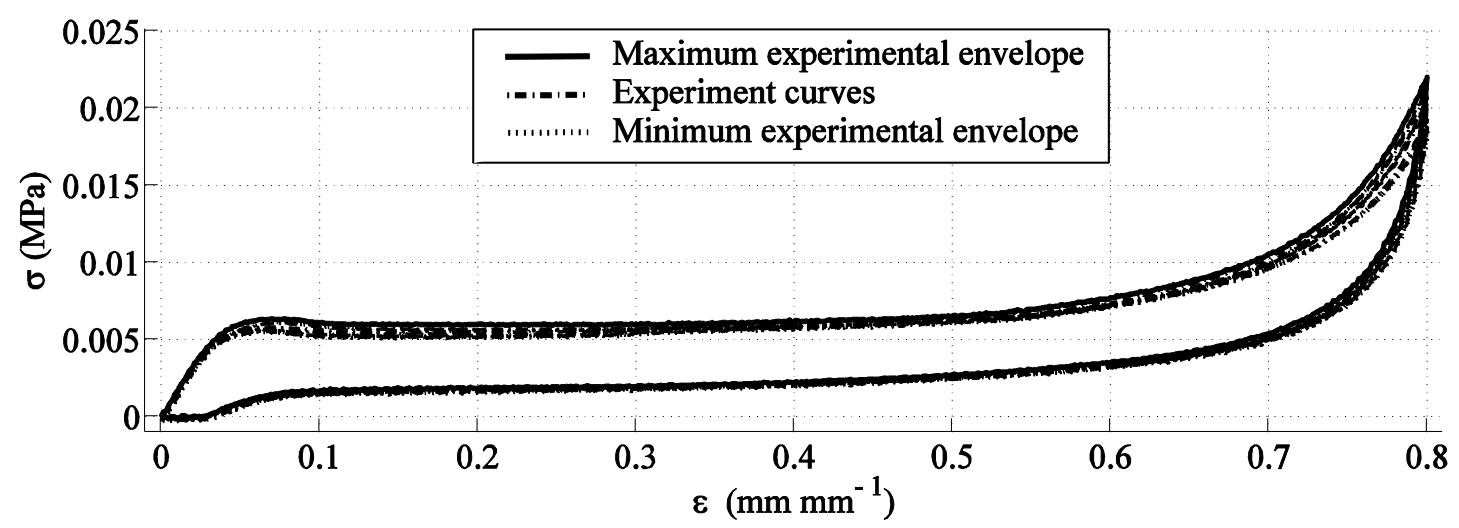

c.

Fig.3. Experimental results for Foam A. a: $\dot{\varepsilon}=1.0610^{-2} \mathrm{sec}^{-1}$; b: $\dot{\varepsilon}=5.3310^{-3} \mathrm{sec}^{-1}$; c: $\dot{\varepsilon}=6.6610^{-4} \mathrm{sec}^{-1}$ 


\subsection{Model results}

The first step was to determine the material parameters which describe the loading process for polyurethane foam using the loading experimental curves of three foams. Then the material parameters which describe the unloading process were determined, based on the experimental unloading stress-strain curve. The loading and unloading parameters were determined using Ogden's model in equation (12), with a three-term expression. The parameter results for three foams in the three tests are given in Table 4.

Table 4. Parameter results for three foams with three strain rates

\begin{tabular}{|c|c|c|c|c|c|c|c|c|c|c|c|}
\hline & & & $\mu_{1}$ & $\alpha_{1}$ & $\beta_{1}$ & $\mu_{2}$ & $\alpha_{2}$ & $\beta_{2}$ & $\mu_{3}$ & $\alpha_{3}$ & $\beta_{3}$ \\
\hline \multirow{6}{*}{$\begin{array}{c}\text { Foam } \\
\text { A }\end{array}$} & \multirow{2}{*}{$\begin{array}{c}\text { Test } \\
1\end{array}$} & load & 0.163 & 55.98 & -0.022 & $2.510^{-3}$ & 24.23 & 0.0612 & $2.0 \mathrm{e}-3$ & 24.22 & 0.054 \\
\hline & & unload & $1.48 \mathrm{e}-8$ & 19.8 & 0.446 & $4.66 \mathrm{e}-3$ & 19.72 & 0.0428 & $9.89 \mathrm{e}-3$ & 19.70 & -0.063 \\
\hline & \multirow{2}{*}{$\begin{array}{c}\text { Test } \\
2\end{array}$} & load & 0.149 & 55.98 & -0.021 & $1.710^{-3}$ & 23.95 & 0.0628 & $1.98 \mathrm{e}-3$ & 23.95 & 0.063 \\
\hline & & unload & $1.38 \mathrm{e}-8$ & 19.8 & 0.448 & $6.35 \mathrm{e}-3$ & 19.71 & 0.0331 & $9.69 \mathrm{e}-3$ & 19.70 & -0.059 \\
\hline & \multirow{2}{*}{$\begin{array}{c}\text { Test } \\
3 \\
\end{array}$} & load & 0.149 & 55.98 & -0.019 & $6.810^{-4}$ & 23.95 & 0.0738 & 0.002 & 23.95 & 0.075 \\
\hline & & unload & $1.22 \mathrm{e}-8$ & 19.8 & 0.449 & $4.96 \mathrm{e}-3$ & 19.70 & 0.0378 & $9.99 \mathrm{e}-3$ & 19.70 & -0.060 \\
\hline \multirow{6}{*}{$\begin{array}{c}\text { Foam } \\
\text { B }\end{array}$} & \multirow{2}{*}{$\begin{array}{c}\text { Test } \\
1\end{array}$} & load & 0.0228 & 55.98 & -0.137 & $2.910^{-3}$ & 23.95 & 0.0472 & 0.0275 & 23.95 & -0.055 \\
\hline & & unload & $7.9 \mathrm{e}-7$ & 19.8 & 0.291 & $1.34 \mathrm{e}-3$ & 19.7 & 0.0296 & $1.34 \mathrm{e}-3$ & 19.7 & 0.0296 \\
\hline & \multirow{2}{*}{$\begin{array}{c}\text { Test } \\
2\end{array}$} & load & 0.0196 & 55.98 & -0.137 & $2.810^{-3}$ & 23.95 & 0.0459 & 0.0251 & 23.95 & -0.054 \\
\hline & & unload & $2.7 \mathrm{e}-11$ & 19.8 & 0.61 & $1.21 \mathrm{e}-3$ & 19.7 & 0.052 & $5.03 \mathrm{e}-4$ & 19.7 & 0.052 \\
\hline & \multirow{2}{*}{$\begin{array}{c}\text { Test } \\
3\end{array}$} & load & 0.0158 & 55.98 & -0.14 & $1.410^{-3}$ & 23.95 & 0.0595 & 0.0178 & 23.95 & -0.046 \\
\hline & & unload & $5.6 \mathrm{e}-7$ & 19.8 & 0.290 & $1.19 \mathrm{e}-3$ & 19.7 & 0.0297 & $1.19 \mathrm{e}-3$ & 19.7 & 0.0297 \\
\hline \multirow{6}{*}{$\begin{array}{c}\text { Foam } \\
\text { C }\end{array}$} & \multirow{2}{*}{$\begin{array}{c}\text { Test } \\
1 \\
\end{array}$} & load & 0.0196 & 55.98 & -0.143 & $1.310^{-3}$ & 23.95 & 0.0721 & 0.0184 & 23.95 & -0.024 \\
\hline & & unload & $1.05 e-6$ & 19.8 & 0.299 & $3.4 \mathrm{e}-3$ & 19.70 & 0.0035 & $3.4 \mathrm{e}-3$ & 19.70 & 0.0035 \\
\hline & \multirow{2}{*}{$\begin{array}{c}\text { Test } \\
2 \\
\end{array}$} & load & 0.0208 & 55.98 & -0.144 & $1.310^{-3}$ & 23.95 & 0.0762 & 0.0173 & 23.95 & $\begin{array}{l}-0.026 \\
\end{array}$ \\
\hline & & unload & $9.8 \mathrm{e}-7$ & 19.8 & 0.304 & $3.0 \mathrm{e}-3$ & 19.7 & 0.0074 & $3.01 \mathrm{e}-3$ & 19.7 & 0.0074 \\
\hline & \multirow{2}{*}{$\begin{array}{c}\text { Test } \\
3\end{array}$} & load & 0.016 & 55.98 & -0.148 & $6.410^{-4}$ & 23.95 & 0.0917 & 0.0118 & 23.95 & -0.015 \\
\hline & & unload & $1.01 \mathrm{e}-6$ & 19.8 & 0.304 & $2.59 \mathrm{e}-3$ & 19.70 & 0.0074 & $2.60 \mathrm{e}-3$ & 19.70 & 0.0074 \\
\hline
\end{tabular}

It can be seen in Table 4 that the values of parameters are different between the loading and unloading phases, which corresponds to the experimental results.

Figure 4 shows the best set of loading-unloading data for three foams in test 1. Figure 5 shows that the model results are in agreement with the experimental results for foam $\mathrm{A}$ in three different tests.

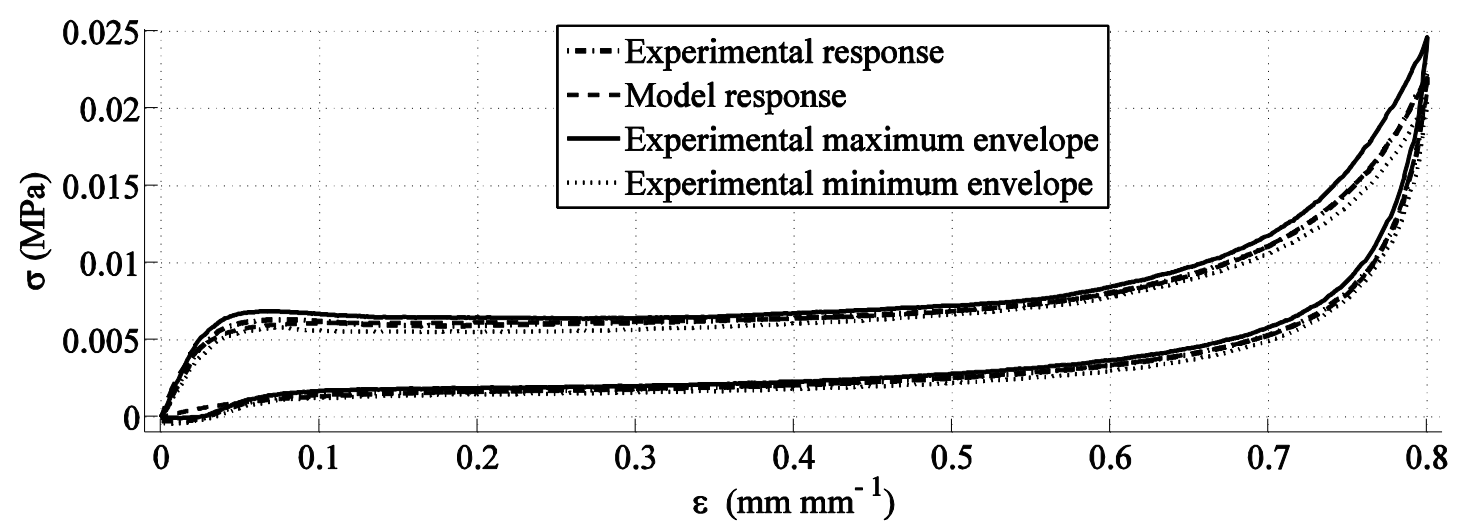

a. 


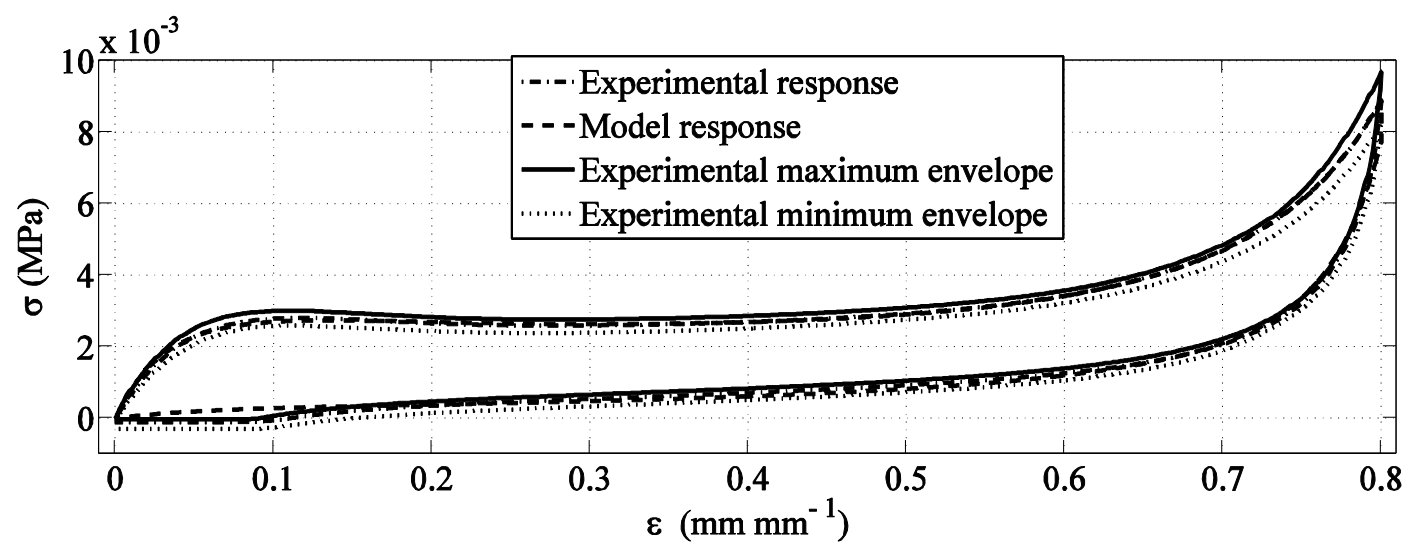

b.

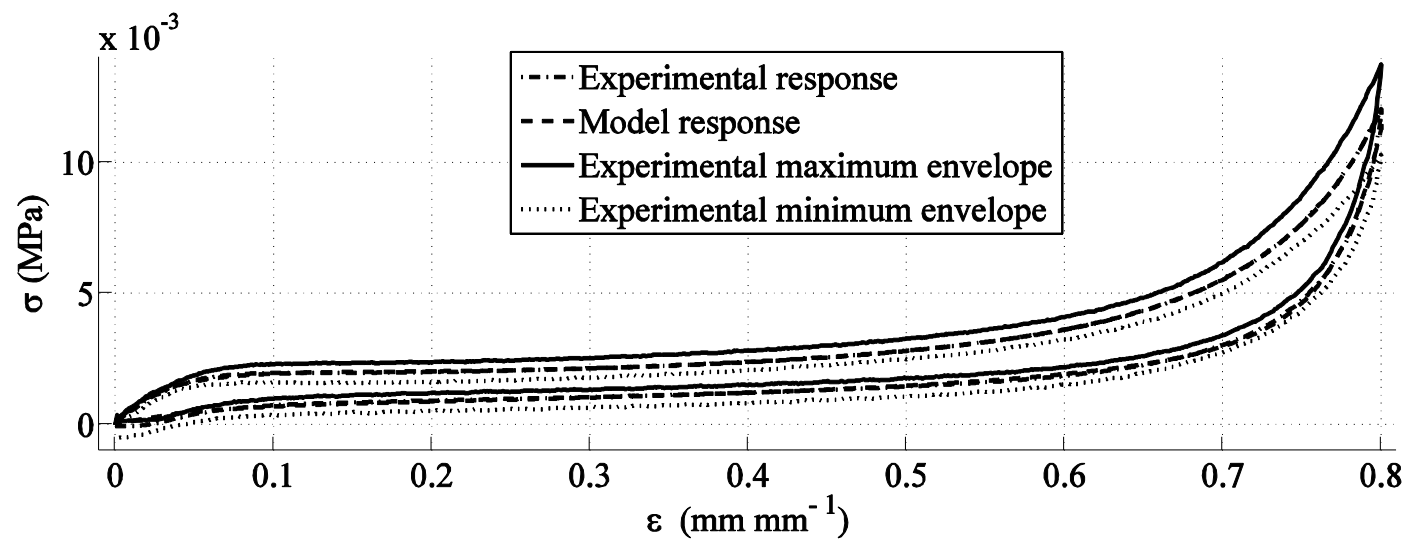

c.

Fig. 4. Model results with strain rate $\dot{\varepsilon}=1.0610^{-2} \sec ^{-1}$ (test1). a: foam A; b: foam B; c: foam C

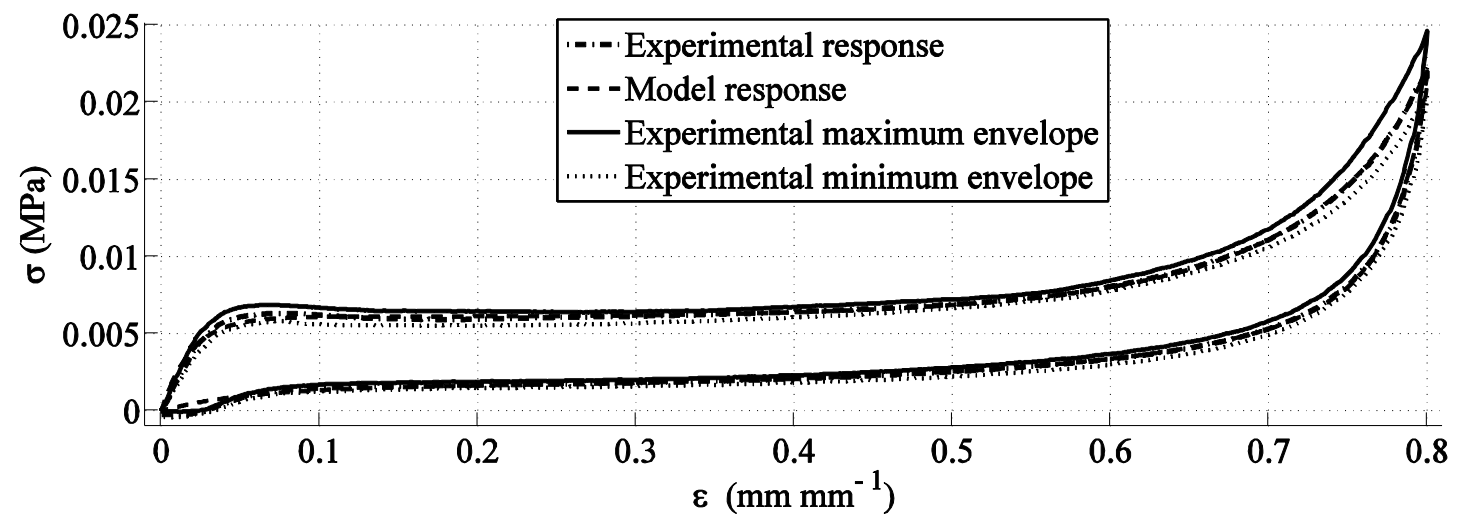

a.

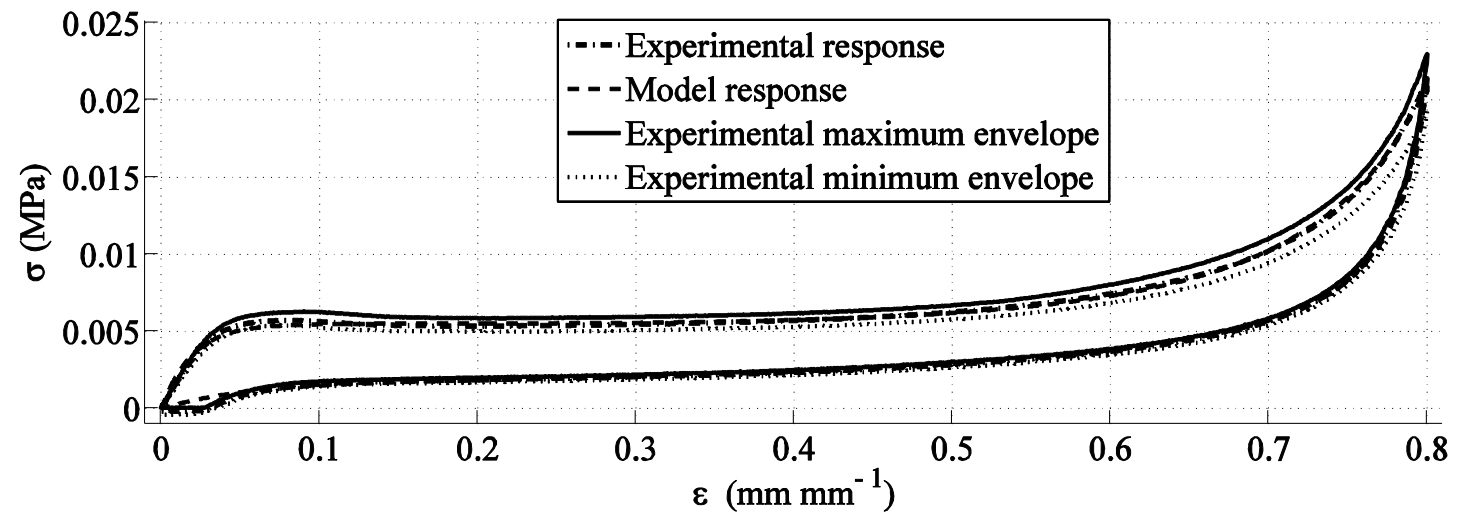

b. 


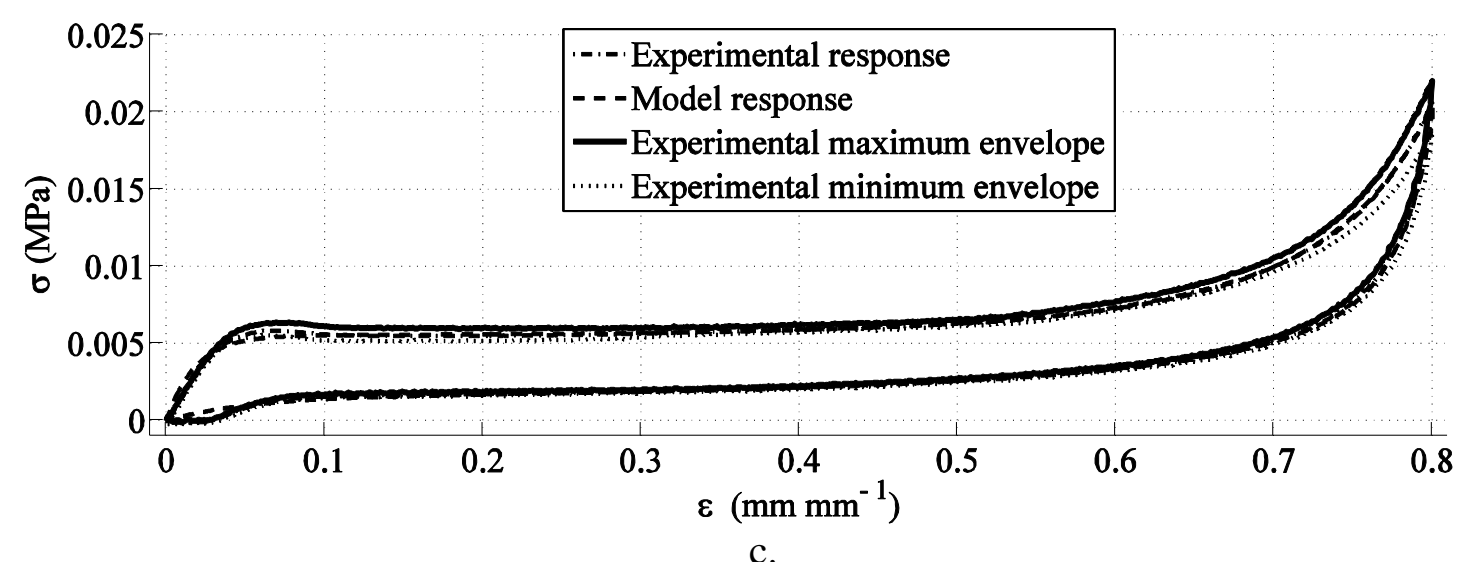

Fig. 5. Model results for foam A. a: $\dot{\varepsilon}=1.0610^{-2} \mathrm{sec}^{-1}$; b: $\dot{\varepsilon}=5.3310^{-3} \mathrm{sec}^{-1}$; $: \dot{\varepsilon}=6.6610^{-4} \mathrm{sec}^{-1}$

\subsection{Discussion}

In Figures 4 and 5, it appears that there is a good correspondence between the Ogden model and the experimental results, especially in the high compression phase. The model curves are between the maximum and minimum envelopes. Table 4 shows that the parameters of the loading phase are different from those of the unloading phase for the unixial compression test and all the parameters are satisfied with the stability conditions of the inequalities (17). In order to ensure the statistical quality of all identified parameters, it is necessary to know the minimum of $\mathrm{N}$ test samples (Table 3). This quality is reviewed through the set at a 95\% confidence level and the statistical limit error "SLE" shall not exceed $10 \%$. To calculate the minimum number of test samples for each strain rate, the following equation is used ${ }^{[1]}$ :

$$
N=\max _{l}\left(1+\operatorname{ceil}\left(\left(\frac{100}{S L E} \frac{u_{l} \hat{\sigma}_{l}}{\hat{m}_{l}}\right)^{2}\right)\right)
$$

where $\hat{m}_{l}$ and $\hat{\sigma}_{l}$ are the estimated average and standard deviation values corresponding to $l^{\text {th }}$ parameter. Ceil is a function to round to the smallest integer not less than $\mathrm{x}$ in MATLAB. These two parameters are obtained from the identification results of the preliminary test with 15 test samples. $u_{l}$ is a coefficient which can be determined with the probability table suitable for the estimated probability law of $l^{\text {th }}$ parameter. The Student law is used for all parameters in this paper. The identification errors for all tests are summarized in Table 5 .

Table 5. Identification errors

\begin{tabular}{|l|l|l|l|l|}
\hline & & Test 1 & Test 2 & Test 3 \\
\hline \multirow{2}{*}{ Foam A } & Load & $1.7 \%$ & $1.9 \%$ & $2.0 \%$ \\
\cline { 2 - 5 } & Unload & $3.4 \%$ & $3.5 \%$ & $3.5 \%$ \\
\hline \multirow{2}{*}{ Foam B } & Load & $1.2 \%$ & $0.9 \%$ & $0.6 \%$ \\
\cline { 2 - 5 } & Unload & $9.1 \%$ & $9.8 \%$ & $9.2 \%$ \\
\hline \multirow{2}{*}{ Foam C } & Load & $0.6 \%$ & $0.7 \%$ & $0.5 \%$ \\
\cline { 2 - 5 } & Unload & $2.9 \%$ & $3.8 \%$ & $5.4 \%$ \\
\hline
\end{tabular}

Table 5 shows that the errors in the unloading phase are always higher than those in the loading phase for each test. In figures 4 and 5, it can also be seen that the experimental and model curves do not have the same trend at the end of the test. At the end of the unloading phase, the residual stress cannot make the foam return to its initial position and the foam section is contactless with the upper frame, so the experimental stress in this part is less accurate, which also accounts for higher errors 
in the unloading phase than in the loading phase. This analysis is validated thanks to another error calculation for foam B's unloading phase without the ending part. Figure 4 (b) shows that there is a great difference between the model results and the experimental results with the strain from 0 to 0.2 in the unloading phase. So only the unloading phase is considered, with the strain from 0.8 to 0.2 , and the results are given in Table 6 and figure 6.

Table 6. Identification errors of unloading phase for Foam B

\begin{tabular}{|l|l|l|l|}
\hline Foam B & Test 1 & Test 2 & Test 3 \\
\hline Unloading phase & $9.1 \%$ & $9.8 \%$ & $9.2 \%$ \\
\hline Unloading phase without ending part & $4 \%$ & $3 \%$ & $4 \%$ \\
\hline
\end{tabular}

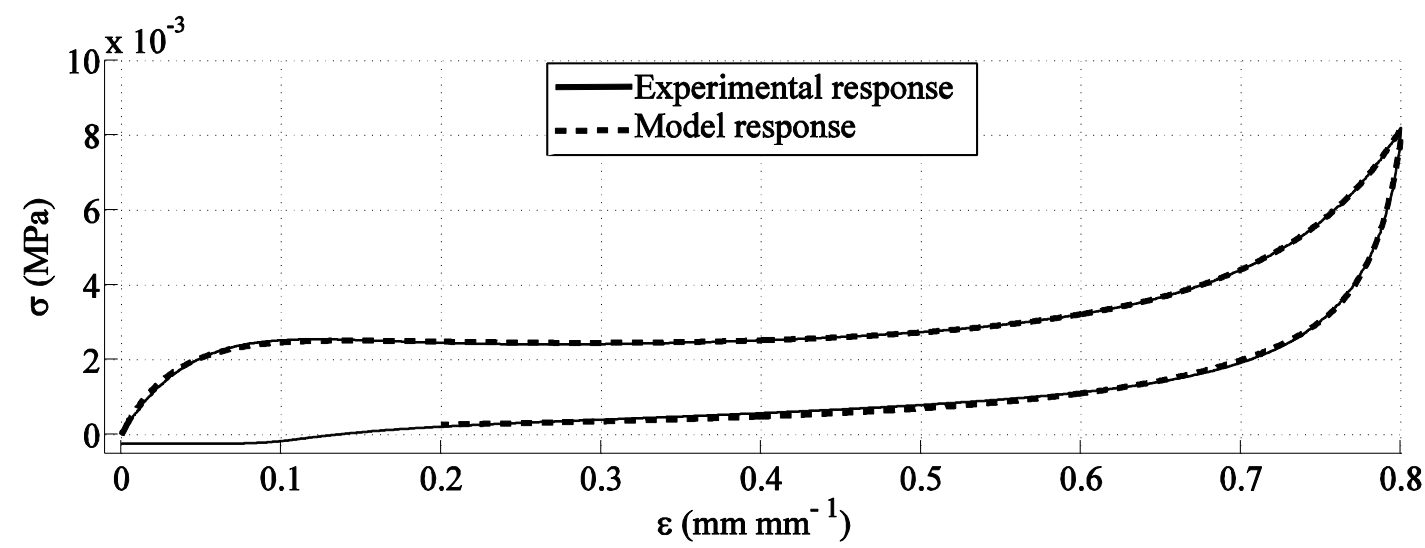

Fig.6. Model results for foam B with strain rate $\dot{\varepsilon}=5.3310^{-3} \mathrm{sec}^{-1}$ in unloading phase without ending part It can be seen in Table 6 that the errors in the unloading phase without the ending part are much smaller than in the whole unloading phase. Figure 6 shows that the unload curve better agrees with the experimental results without the ending part. So the conclusion is that the unloading phase is more sensitive than the loading phase because of the residual stress which has a great effect at the end of the test. The residual strain will slowly decrease in time. After a sufficiently large period, the foams will return to the original configuration. This phenomenon is due to the viscoelasticity behaviour of polyurethane foam which will be discussed in detail in the next paper.

\section{Conclusion}

This paper has presented numerous experiments with three different polyurethane foams in three different strain rates for loading-unloading uniaxial compression tests. The Ogden strain energy density function for compressible materials has been applied to predict the foams mechanical behaviour. The model consists of an incompressibility component and a compressibility component and the stress has been derived from the model in terms of principal stretches. The stability conditions of the Ogden model have been proposed. Several experimental data for compressible polyurethane foam have been used to identify the parameters of the model. The predictions based on the proposed strain energy function compare very well with the experimental data. The errors between the Ogden model and experimental data have been calculated. The results show that the Ogden model can predict the quasi-static mechanical behaviour of polyurethane foam under large strain compression. The model results agree with the experimental results and a detail of the unloading phase in the compression test has been discussed. For further studies, other models will be used and compared with Ogden's model for polyurethane foams and the viscoelasticity behaviour will be presented in a future paper.

\section{Acknowledgements}

The authors wish to thank the China Scholarship Council (CSC) for its financial support. 


\section{References}

[1] Jmal H, Dupuis R, Aubry E. Journal of Cellular Plastics. 47. (2011). 447-465.

[2] Peyraut F, Feng ZQ, He QC, Labed N. Applied Numerical Mathematics. 59. (2009). 14991514.

[3] Romero PA, Zheng SF, Cuitino AM. Journal of the Mechanics and Physics of Solids. 56. (2008). 1916-1943.

[4] Singh R. Dynamic modeling of polyurethane foam and development of system identification methodologies. In: School of Mechanical Engineering West Lafayette: Purdue University, (2000).

[5] Gibson LJ, Ashby MF. Cellular Solids:Structure and Properties. Cambridge, U.K.: Cambridge University Press, (1997).

[6] Mooney M. Journal of Applied Physics. 11. (1940). 582-592.

[7] Treloar LRG. Clarendon Press. (1975).

[8] Rivlin RS. Philosophical Transitions of the Royal Society of London. A 240. (1948). 459-490.

[9] Rivlin RS. Proceedings of the Cambridge Philosophical Society. 45. (1949). 485-487.

[10] Yang LM, Shim VPW. International Journal of Impact Engineering. 30. (2004). 1099-1110.

[11] Blatz PJ, Ko WL. Transactions of the Society of Rheology. 6. (1962). 223-251.

[12] Ogden RW. Proceedings of the Royal Society of London. 326. (1972). 565-584.

[13] Ogden RW. Proceedings of the Royal Society of London. 328. (1972). 567-583.

[14] Yeoh OH. Rubber Chemistry and Technology. 66. (1993). 754-771.

[15] Beatty MF. Applied Mechanics Review. 40. (1987). 1699-1734.

[16] Arruda EM, Boyce MC. Journal of the Mechanics and Physics of Solids. 41. (1993). 389-412.

[17] Bischoff JE, Arruda EM, Grosh K. Rubber Chemistry and Technology. (2000). 541-559.

[18] Attard MM. International Journal of Solids and Structures. 40. (2003). 4353-4378.

[19] Sussman T, Bathe K-Jr. Computers and Structures. 26. (1987). 357-409.

[20] Smardzewski J, Grbac I, Prekrat S. DRVNA INDUSTRIJA. 59. (2008). 23-28.

[21] Zhang XF, Andrieux F, Sun DZ. Materials \& Design. 32. (2010). 877-884.

[22] Belouettar S, Rauchs G, Kirsch A, Bieck W. Matériaux \& Techniques 94. (2006). 217-226.

[23] White SW, Kim SK, Bajaj AK, Davies P, Showers DK, Liedtke PE. Nonlinear Dynamics. 22. (2000). 281-313.

[24] Dupuis R, Aubry E. SEM XI International Congress \& Exposition on Experimental \& Applied Mechanics (2008).

[25] Schrodt M, Benderoth G, Kühhorn A, Silber G. TECHNISCHE MECHANIK. (2005). 162173.

[26] Dorfmann A, Ogden RW. International Journal of Solids and Structures. 41. (2004). 18551878.

[27] Green AE, Adkins JE. Large Elastic Deformations Oxford: Oxford University Press, (1970).

[28] Shen Y, Golnaraghi F, Plumtree A. International Journal of Fatigue. 23. (2001). 491-497.

[29] Hill R, Chia-Shun Y. Aspects of Invariance in Solid Mechanics. In: Advances in Applied Mechanics, Vol. Volume 18: Elsevier, 1979, pp. 1-75.

[30] Storakers B. Journal of the Mechanics and Physics of Solids. 34. (1986). 125-145. 